\title{
APLICACIONES DE LAS REDES DE PETRI EN LA CONSTRUCCIÓN
}

\section{(APPLICATIONS OF PETRI NETS IN CONSTRUCTION)}

\author{
Alfonso Recuero, Dr. Ing. de Caminos \\ Instituto Eduardo Torroja-CSIC \\ Marina Álvarez, Dra. Arquitecta \\ Facultad de Informática, UPM, Campus de Monte Gancedo \\ Boadilla del Monte, Madrid
}

ESPAÑA

Fecha de recepción: 29-X-96

403-19

\section{RESUMEN}

Las redes de Petri son una herramienta muy importante para el análisis de sistemas dinámicos, herramienta que debiera ser conocida por cualquier experto en ciencias de la computación. Dentro del campo de la construcción, sus aplicaciones más claras están en la fabricación de materiales y elementos $y$ en el intercambio de mensajes electrónicos entre los distintos participantes en el proceso constructivo.

En este trabajo se presenta una sucinta descripcion de las redes de Petri, mostrando su aplicación al intercambio de mensajes.

\section{SUMMARY}

Petri nets is a very important tool for the analysis of dynamic systems. It should be know by all experts in computational science. Their most important applications in building are in fabrication processes an in the exchange of electronic messages among the different participants in the building process.

In this work, a brief description of Petri nets is presented, and their application to message interchange is shown.

\section{Introducción}

El proceso constructivo consiste en una secuencia de actividades (proyecto arquitectónico, estructural, de instalaciones, planificación de la obra, ejecución de la misma, etc.) desarrollada por distintos participantes (arquitectos, ingenieros, constructores, etc.), con objeto de crear un nuevo producto o modificar uno existente, algunas de las cuales tienen lugar de forma concurrente. Esta descripción, válida para otros tipos de industria, tiene algunas características específicas en la construcción, tales como:

-Habitualmente hay más de un participante involucrado en el proyecto y construcción de un edificio.

-Los participantes varían de un edificio a otro.

-La mayor parte de los participantes son empresas pequeñas o medianas.

-Los productos se producen en cantidades reducidas.

Describir este sistema es una tarea que consiste en la elaboración de un modelo del mismo. Con este fin se pueden utilizar técnicas de la ciencia de la computación

como son las redes de Petri, herramienta de descripción que conjuga las posibilidades gráficas y las analíticas y que es fácilmente comprensible por el futuro usuario del sistemay, mediante la cual, es posible formalizar su diálogo con el diseñador y otros participantes del proceso. Las redes de Petri se han utilizado en diferentes campos de aplicación, desde los sistemas legales, sistemas operativos y descripción del software y hardware en general, los autómatas lógicos, lenguajes formales, a la concepción de mecanismos de asignación de recursos y de procedimientos de sincronización de tareas en sistemas centralizados o repartidos, así como para la elaboracion de cuadernos de carga de sistemas de control de procesos industriales.

Es importante señalar la ventaja que tiene la utilización de representaciones gráficas adecuadas para comprender, más fácilmente, aspectos de los sistemas dinámicos, tales como la dependencia causal, secuencias de operaciones, conflictos en el uso de recursos, concurrencia, etc.

Dentro de la construcción se utilizan -desde hace algunos años-en la fabricación de materiales y en la prefabricación 
de elementos. Es importante considerar que, como toda actividad de diseño, la utilidad de las redes de Petri depende de la metodología con que se emplee, así como de la disponibilidad de sistemas automáticos de ayuda, CAD, etc.

Este trabajo es parte del Proyecto de Investigación "Desarrollo, mejora e integración de sistemas $\mathrm{CAD} / \mathrm{CAE}$ en Construcción", que se lleva a cabo en el Instituto Eduardo Torroja- CSIC, financiado por la DGICYT (PB 930111).

\section{Redes de Petri}

Las redes de Petri se han desarrollado a partir de los trabajos de Carl Adam Petri en su tesis doctoral "Kommunikation mit Automaten", presentada en 1962. En ella formulaba las bases de una teoría de comunicación entre componentes asíncronos de un sistema informático, en la que prestaba especial atención a la descripción de relaciones causales entre sucesos. Desde este momento, la teoría de las redes de Petri ha tenido un desarrollo considerable. Sin embargo, la mayor parte de estos desarrollos sólo están disponibles en forma de informes internos, tesis doctorales o en presentaciones a congresos, pese a lo cual, su uso está cada vez más extendido. De hecho, se considera que es una herramienta que debe ser conocida por cualquier investigador en ciencias de la computación.

Existen libros que abordan el tema de forma organizada y didáctica (Brahms, Silva, Peterson, Reisig, etc.), así como actos de conferencias anuales dedicadas específicamente al tema (Jensen).

Una red de Petri es un grafo orientado en el que intervienen dos clases de nudos, las "plazas" o "lugares" (representadas por circunferencias) y las "transiciones" (representa- das por rectángulos o barras), unidos, alternativamente, por arcos valorados. Cuando todos los arcos son unitarios se dice que la red es no valorada. Un arco une una plaza con una transición, o viceversa, pero nunca dos transiciones o dos plazas. Esto constituye la parte estructural o fija de la red.

Una plaza puede contener un número entero, positivo o nulo, de marcas. Una marca se representa por un punto en el interior del círculo correspondiente a la plaza. El conjunto de marcas asociadas en un instante dado a cada una de las plazas constituye un marcado de la red. La evolución de estos marcados conforma el funcionamiento de la red, permitiendo, de este modo, representar sistemas dinámicos mediante redes de Petri.

Las redes de Petri se utilizan con el objeto de modelizar el comportamiento dinámico de sistemas discretos. El conjunto de plazas permite representar los estados del sistema, siendo el conjunto de transiciones el que representa el conjunto de sucesos, cuya aparición provoca la modificación de los estados del sistema. Más todavía: las plazas juegan el papel de variables de estado. De forma figurada, a cada plaza se le asigna el mismo número de marcas que su correspondiente valor entero. Así, pues, a un estado del sistema le corresponde un marcado que define el número de marcas asociadas a cada plaza (o contenidas en ella).

A la aparición de un suceso, ligado a condiciones externas a la red y que depende del cumplimiento de unas precondiciones, le corresponde el franqueo o disparo de una transición. Estas precondiciones se refieren al número de marcas contenidas en aquellas plazas que están unidas a la transición asociada al suceso considerado. El franqueo de una transición tiene como efecto modificar el marcado: se quitan todas las marcas que han satisfecho la precondi-

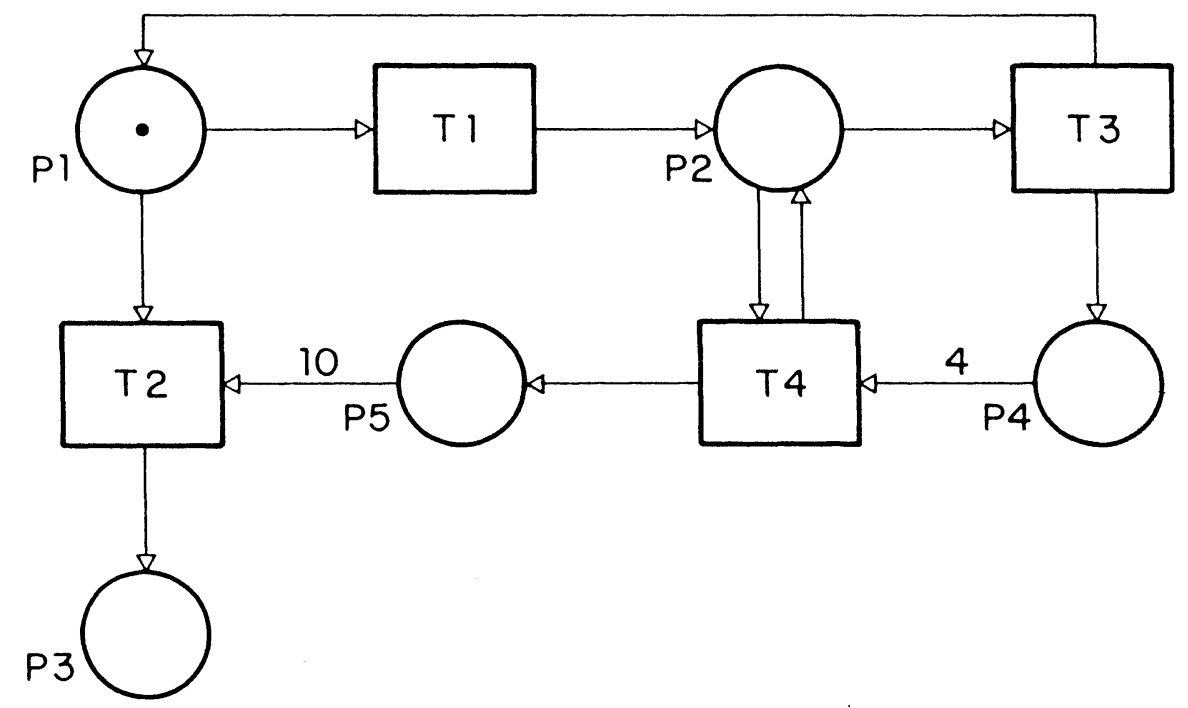

Fig. 1 
ción correspondiente a la transición y se añaden marcas a las plazas de salida de la transición. Debe resaltarse que el cumplimiento de la precondición para una transición no implica que ésta deba franquearse; es preciso, además, que se produzca el suceso exterior.

Mediante una red de Petri puede modelizarse un sistema de evolución en paralelo, compuesto de varios procesos (o agentes) que cooperan para la realización de un objetivo común. En general, la presencia de marcas en una plaza se interpreta como la presencia de recursos de un cierto tipo. El franqueo de una transición representa una acción, que puede tener lugar cuando está disponible un número suficiente de recursos, es decir: cuando se satisfacen las precondiciones. La acción emplea estos recursos para producir otros que son depositados en las plazas de salida.

La red de la Figura 1 es un ejemplo sencillo. Puede interpretarse como se indica a continuación:

P1 puede ser un director de fábrica, $\mathrm{P} 2$ un jefe de producción, P4 un almacén de elementos simples, P5 un almacén de elementos empaquetados de 4 en 4 , y P3 un embarcadero, donde se despachan los paquetes de 10 en 10.

Mediante T1, P1 ordena a P2 que actúe, bien fabricando un nuevo elemento (franqueando T3), que deposita en P4, al tiempo que se lo comunica a P1 y queda en espera de órdenes, o bien empaquetando, de 4 en 4 , los elementos almacenados en $\mathrm{P} 4$; siempre que haya suficientes elementos y almacenando los paquetes en P5 (mediante el franqueo de T4). Cuando haya suficientes paquetes en P5, P1 puede decidir (franqueando T2) hacer un embarque de 10, lo que con la red actual provoca el bloqueo del proceso.

Las redes de valoración 1 fueron, históricamente, las primeras que se introdujeron y las realizables más fácilmente. Una red cualquiera puede simularse por una de estas redesy, recíprocamente, cualquier red puede obtenerse por simplificación de una red de valoración 1 . Las redes generales permiten una modelización más natural y más concisa.

Veamos ahora algunos casos simplificados de interés de redes de Petri.

Una máquina de estados es una red de Petri en la que toda transición está unida, exactamente, a una plaza de entrada y a una plaza de salida. Las máquinas de estados modelizan, de forma adecuada, los autómatas de estados finitos e incluso los procesos secuenciales. Si una plaza de entrada de un conjunto de transiciones está marcada, entonces todas ellas son franqueables. Si el marcado inicial tiene una sola marca, ésta se desplaza de plaza en plaza y designa el estado en curso del autómata (en el caso de un programa, señala la siguiente instrucción a ejecutar). Si se admiten varias marcas, éstas se desplazan de forma completamente independiente (simulando así un conjunto de procesos independientes que tienen el mismo programa). Sin embargo, las máquinas de estados no permiten, por sí solas, la expresión de sincronizaciones entre procesos. La Figura 2 muestra un ejemplo de máquina de estados.

Un grafo de sucesos es una red de Petri en la que toda plaza está unida exactamente a una transición de entrada y a una transición de salida. Los grafos de sucesos modelizan, de forma adecuada, los sistemas de ordenación de tareas. Estas tareas cooperan sin conflicto, transmitiéndose resultados. Puede haber reciclaje, lo que proporciona una mayor generalidad que las redes PERT clásicas. Sin embargo, los grafos de sucesos no permiten modelizar ni elecciones ni conflictos de asignación de recursos entre procesos. La Figura 3 muestra un ejemplo de grafo de sucesos.

Por último, si una red es, a la vez, una máquina de estados y un grafo de sucesos, entonces se reduce a un conjunto de circuitos disjuntos.

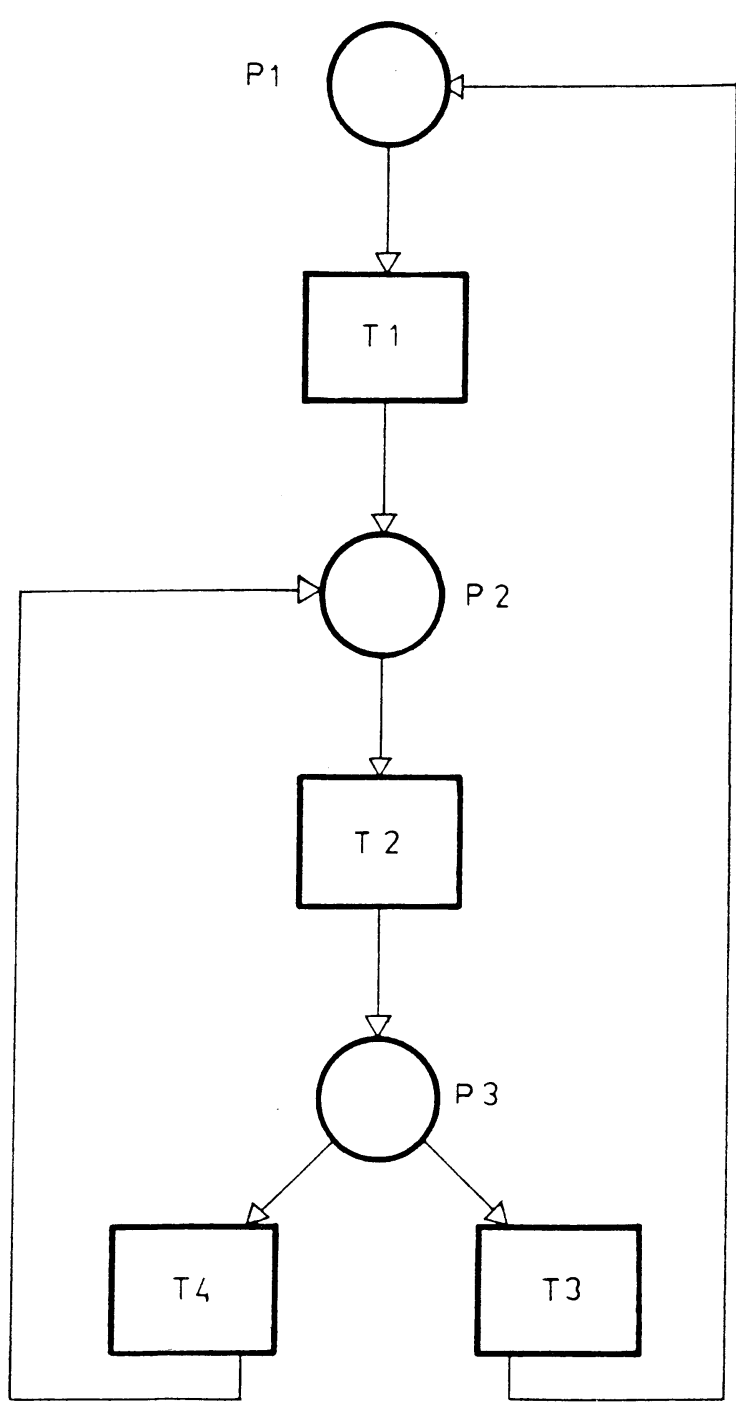

Fig. 2 


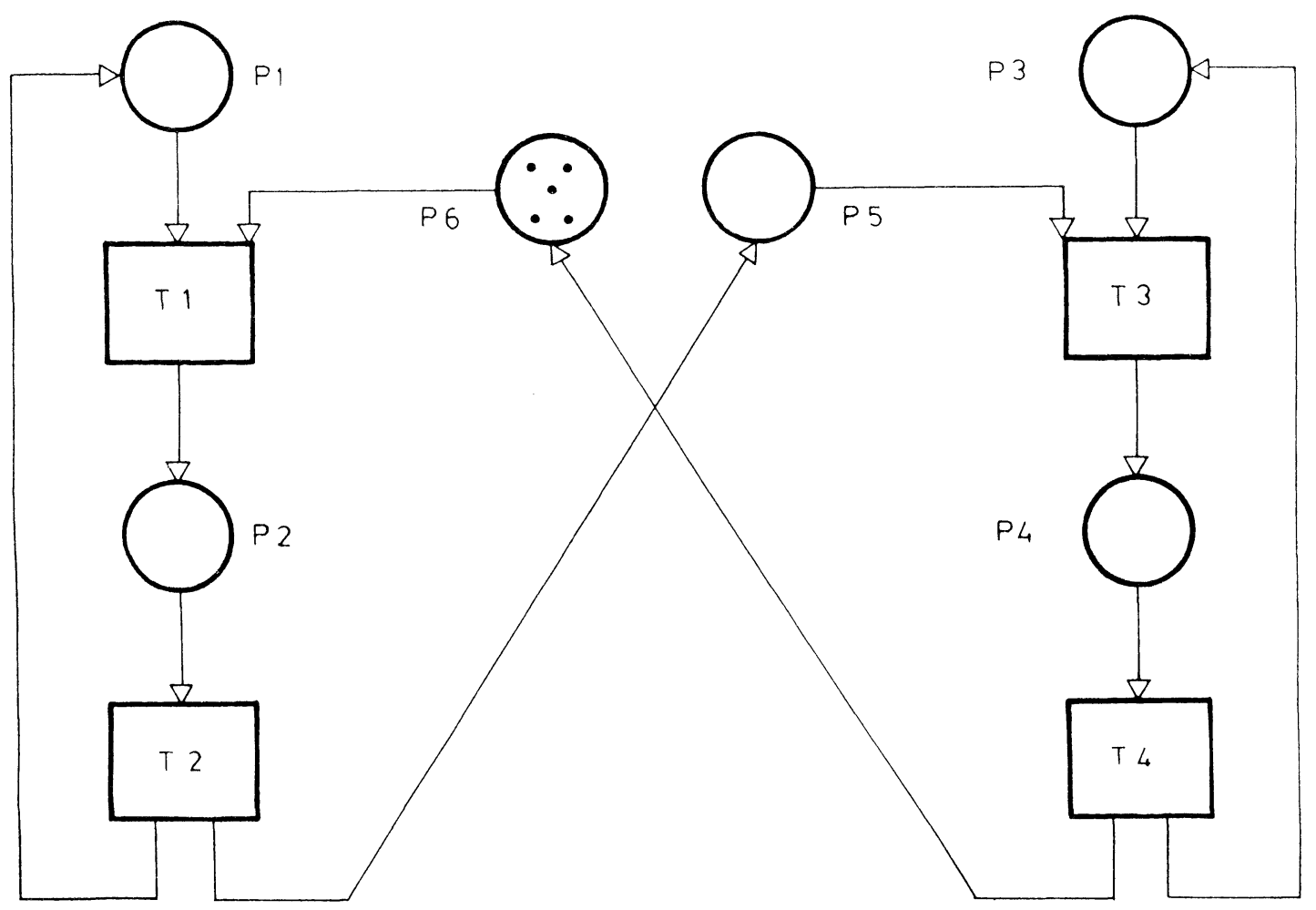

Fig. 3

\section{Propiedades de las redes de Petri}

Se presenta un conjunto de subrutinas, escritas en Qbasic, que permiten el análisis de una red de Petri. Se ha elegido un lenguaje de programación de fácil comprensión, empleando técnicas de programación estructurada, lo que permite su trascripción a otros lenguajes de forma simple. Se ha elegido un esquema de almacenamiento de datos compacto, pues sólo se almacenan los valores significativos, resultando muy eficiente a la hora de manejarlos. Mediante el uso de dichas subrutinas pueden analizarse las principales propiedades de las redes de Petri, que aquí, solamente, se van a enunciar.

Distinguiremos dos tipos de propiedades: aquéllas que dependen sólo de la estructura de la red y aquéllas que se relacionan con su funcionamiento.

Una red se denomina "pura" cuando ninguna transición tiene arcos de entrada y de salida con una misma plaza. Para comprobar la pureza de una red bastará comprobar este extremo, revisando su estructura.

Se dice que la red presenta conflictos estructurales cuando existen transiciones que comparten alguna plaza de entrada. Nuevamente, basta con revisar la estructura de la red para comprobar su existencia.

La herramienta elegida para el análisis de las propiedades dependientes del funcionamiento de una red de Petri es el llamadoárbol de cobertura. Cada uno de los nodos del árbol de cobertura representa un marcado accesible y cada arco la transición que permite pasar de un nodo padre a uno descendiente. En el primer nivel se coloca el marcado inicial, procediéndose a generar el árbol en anchura. Para ello se recorren todos los nodos de un nivel, se comprueba que no son terminales y se generan todos los nodos descendientes, franqueando todas las transiciones accesibles desde ese marcado. Cuando no se genera ningún nodo de un nuevo nivel, el árbol de cobertura queda completado.

Cuando a un nodo del árbol de cobertura le corresponde un marcado mayor que el correspondiente a un nodo antecedente (en la misma rama), las plazas del mismo, con marca mayor que la del antecedente, no están acotadas, puesto que la secuencia de transiciones que los une puede repetirse indefinidamente. Esto daría lugar a un árbol infinito, para evitarlo, se sustituye la marca de dichas plazas, en el marcado mayor, por un valor $\mathrm{W}$, que no es afectado por el franqueo de transiciones. La Figura 4 muestra el árbol de cobertura correspondiente al ejemplo de la Figura 1.

Cuando se alcanza un marcado que no permite franquear ninguna transición, se ha alcanzado una situación de bloqueo. Se denominan secuencias de bloqueo a las secuencias de transiciones que permiten pasar del marcado inicial a la situación de bloqueo.

Cuando en una misma rama del árbol de cobertura aparecen dos marcados iguales, la secuencia de franqueos que 
permite pasar de uno a otro puede repetirse indefinidamente. La convención utilizada para marcar las plazas no acotadas permite detectar también las secuencias que, en caso de repetirse indefinidamente, conducen a marcados infinitos en dichas plazas. A estas secuencias de franqueos se las denomina secuencias repetitivas.

Una transición se dice cuasi-viva, para un marcado inicial, cuando es posible franquearla, al menos, una vez. De no ser así, la transición sería inútil para dicho marcado. Si todas las transiciones son cuasi-vivas, la red se dice que es cuasiviva. Evidentemente, una red no puede ser cuasi-viva para



Fig. 4 cualquier marcado, pudiéndose encontrar -siempre también- un marcado para el que là red sea cuasi-viva. Más importante es el concepto de vivacidad. Se dice que una red es viva para un marcado inicial, cuando es cuasi-viva para cualquier marcado accesible a partir del inicial.

\section{Análisis del ejemplo de la Figura 1}

La red de la Figura 1 es un ejemplo sencillo, pero a la vez ilustrativo, ya que permite ver la aplicación de todas las subrutinas. Los resultados del análisis se muestran a continuación.

5 Plazas, 4 transiciones, valorada 1

T1 IN: 1, 1\ OUT: 2, $1 \backslash$

T2 IN: $1,1 \backslash 5,10 \backslash$ OUT: $3,1 \backslash$

T3 IN: 2, $1 \backslash$ OUT: $1,1 \backslash 4,1 \backslash$

T4 IN: $2,1 \backslash 4,4 \backslash$ OUT: $2,1 \backslash 5,1 \backslash$

Pureza de la red

LA TRANSICIÓN 4 PRODUCE UN BUCLE EN LA PLAZA 2 Conflictos estructurales

LAS TRANSICIONES 1 Y 2 COMPARTEN LA PLAZA DE ENTRADA 1

LAS TRANSICIONES 3 Y 4 COMPARTEN LA PLAZA DE ENTRADA 2

Generación del árbol de cobertura

M1 $(\mathrm{T} 1>\mathrm{M} 2$ NODO $1 \rightarrow 2$

$\mathrm{M} 2(\mathrm{~T} 3>\mathrm{M} 3$ NODO $2 \rightarrow 3$

$\mathrm{M} 3(\mathrm{~T} 1>\mathrm{M} 4$ NODO $3 \rightarrow 4$

$\mathrm{M} 4$ (T3 $>$ M3 NODO $4 \longrightarrow 5$ REPR. UNMARCADO ANTERIOR

M4 (T4 $>$ M5 NODO $4 \longrightarrow 6$

M5 $($ T3 $>$ M6 NODO $6 \rightarrow 7$

M5 (T4 $>$ M5 NODO $6 \longrightarrow 8$ REPR. UNMARCADO ANTERIOR M6 (T1 $>$ M5 NODO $7 \rightarrow 9$ REPR. UNMARCADO ANTERIOR M6 $($ T2 $>$ M7 NODO $7 \rightarrow 10$

Marcados alcanzados

$\mathrm{M} 1: \mathrm{P} 1=1$

$\mathrm{M} 2: \mathrm{P} 2=1$

$\mathrm{M} 3: \mathrm{P} 1=1 \quad \mathrm{P} 4=\mathrm{W}$

$\mathrm{M} 4: \mathrm{P} 2=1 \quad \mathrm{P} 4=\mathrm{W}$

$\mathrm{M} 5: \mathrm{P} 2=1 \quad \mathrm{P} 4=\mathrm{W} \quad \mathrm{P} 5=\mathrm{W}$

$\mathrm{M} 6: \mathrm{P} 1=1 \quad \mathrm{P} 4=\mathrm{W} \quad \mathrm{P} 5=\mathrm{W}$

$\mathrm{M} 7: \mathrm{P} 3=1 \quad \mathrm{P} 4=\mathrm{W} \quad \mathrm{P} 5=\mathrm{W}$

Secuencias repetitivas

$\mathrm{S} 1: \mathrm{Tl}>\mathrm{T} 3>$

$\mathrm{S} 2: \mathrm{T} 4>$

S3: T3> T1>

Secuencias de bloqueo

$\mathrm{S} 1$ : $\mathrm{M} 1$ (T1> M2 (T3> M3 (T1>M4 (T4> M5 (T3>M6 (T2>M7

Vivacidad de la red, para el marcado inicial

LA RED ES CUASI-VIVA PARA EL MARCADO INICIAL

T1 NO ES ACCESIBLE DESDE M7

T2 NO ES ACCESIBLE DESDE M7

T3 NO ES ACCESIBLE DESDE M7

T4 NO ES ACCESIBLE DESDE M7 
Si se agregase un arco unitario de salida de T3 a P1, el proceso no se bloquearía al realizar un embarque. Cuando se vean las posibles ampliaciones a las redes de Petri básicas, se indicarán otras formas de interés para modificar el funcionamiento de la red del ejemplo

\section{Extensiones de las redes de Petri}

Hasta aquí se ha tratado de las redes de Petri en sentido estricto. Numerosos autores han propuesto distintas extensiones que permiten mejorar su funcionamiento, permitiéndolas representar sistemas que no pueden ser modelados por medio de redes de Petri estrictas. A continuación se describen algunas de estas extensiones, concretamente la utilización de arcos inhibidores, la priorización de transiciones en conflicto, las redes coloreadas y las redes temporizadas.

\section{Utilización de arcos inhibidores}

Cuando se desea que una transición $\mathrm{T}$ tenga como condición para ser franqueada que la marca de una plaza $P$ sea 0 , puede utilizarse un arco inhibidor que conecte a $\mathrm{P}$ como plaza de entrada de $\mathrm{T}$. De forma más general, pueden establecerse arcos inhibidores condicionados a que la marca tenga un valor igual o menor que uno concreto que no se modifique por el franqueo de $\mathrm{T}$.

El esquema utilizado permite representar este tipo de arcos. Para ello, la red debe ser valorada, e incluir el arco inhibidor (con el valor de marca máxima con signo negativo) como arco de entrada de $\mathrm{T}$. Llamaremos plazas inhibidoras a aquéllas que son origen de un arco inhibidor. No se debe aplicar a tales plazas el criterio adoptado para las plazas no acotadas, pues hay que hacer en ellas comprobaciones sobre el número exacto de marcas que contienen.
Si en el ejemplo de la Figura 1 deseásemos limitar a 4 elementos la capacidad del almacén en P4, haciendo que fuesen empaquetados al alcanzar dicho número, y cuando tuviésemos 10 paquetes en P5 fuesen embarcados antes de recibir nuevos paquetes, podríamos introducir un arco inhibidor, con valor -3 , de P4 a T3 y otro, de valor -9 , de P5 a T1, tal como se muestra en la red de la Figura 5, en la que los arcos inhibidores se dibujan como una línea continua, con un pequeño círculo en el extremo de llegada a la transición. Sin embargo, al analizar esta red se detecta un problema. Cuando se alcanza la situación de tener 10 paquetes en P5, para que P1 pueda dar la orden de embarcarlos (franqueo de T2), ha de estar marcada. Para ello ha de franquearse T3, lo que deposita un elemento en P4.

Una generalización más amplia de este tipo es la propuesta por Vala (self modifying nets), en la que las reglas de franqueo pueden modificarse, de modo que una transición quita o pone un número de marcas, dependiendo del contenido de ciertas plazas.

\section{Redes priorizadas}

Cuando una red presenta conflictos estructurales, éstos pueden convertirse en conflictos efectivos para ciertos marcados. Esto es, cuando una plaza es de entrada para más de una transición, puede ocurrir que el número de marcas de la plaza no sea suficiente para permitir el franqueo simultáneo de todas ellas. Tales situaciones pueden resolverse estableciendo prioridades de franqueo entre las transiciones en conflicto.

Así, si en el ejemplo estableciésemos que la transición T4 tiene preferencia sobre la T3 y que la T2 la tiene sobre la $\mathrm{T} 1$, se producirían cambios en su funcionamiento -análo-

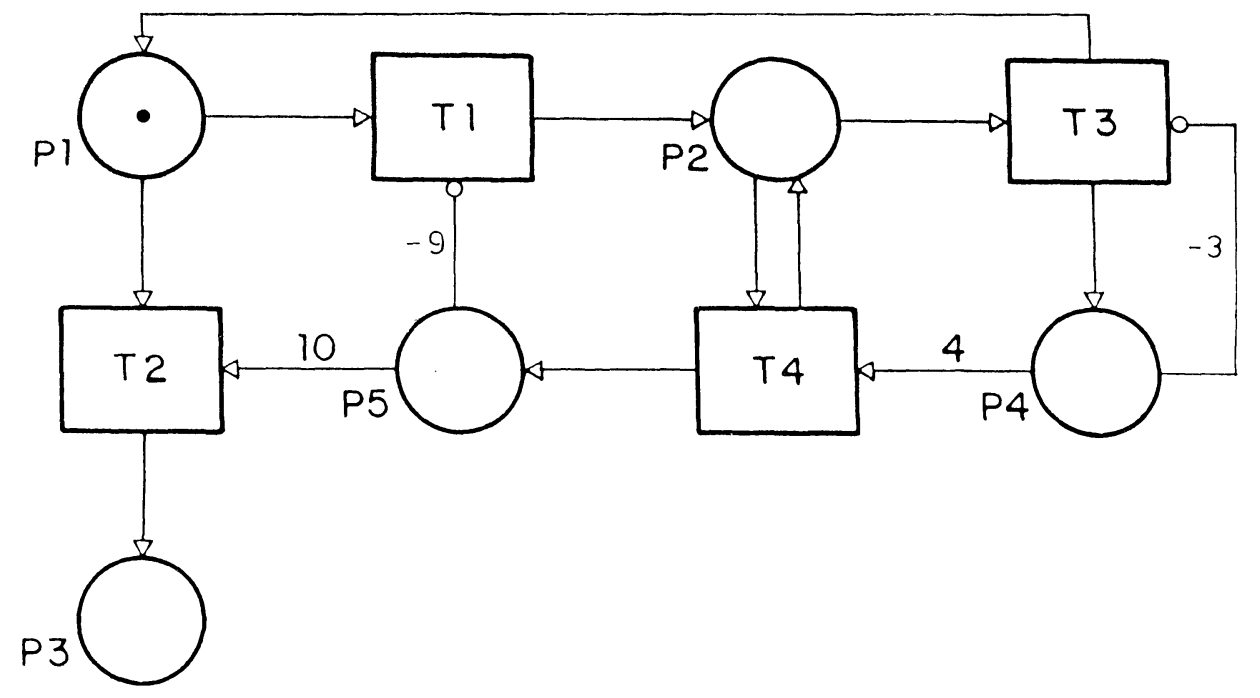


gos a los descritos en el apartado anterior- mediante la introducción de arcos inhibidores. El esquema propuesto permite considerar este tipo de extensión.

\section{Redes coloreadas}

Se han propuesto otros tipos de extensiones que afectan al contenido de las plazas o al modo de franqueo de las transiciones. Las plazas, en lugar de un número, pueden contener objetos con una estructura más rica, tal como una lista, o pila, o distinguirlas mediante un "coloreado", pudiendo las transiciones modificar estos objetos con reglas adaptadas

Las redes de Petri coloreadas han sido introducidas para condensar la descripción de sistemas en los que se identifican diversos subsistemas con estructura y comportamiento similares, pero que trabajan en paralelo. En una red de Petri coloreada, cada marca puede portar un color que la identifique.

A cada plaza y a cada transición se le asigna un conjunto de colores. Una transición puede franquearse respecto a cada uno de sus colores. El franqueo de una transición elimina y añade marcas como en las redes normales, pero respetando la dependencia funcional especificada entre el color del franqueo de la transición y los colores de las marcas. El color de cada marca puede ser cambiado por el franqueo de una transición.

\section{Redes temporizadas}

Otro tipo de extensiones son aquéllas en las que el funcionamiento de la red depende del entorno y no sólo de las condiciones propias de la red (redes no autónomas). Puesto que las redes son un modelo para la descripción de un flujo de control, puede ser necesario aumentar y enriquecer este modelo cuando se quiere utilizar para describir programas o sistemas paralelos. Con esta finalidad, se añade un entorno formado por un conjunto de variables y de operadores que "dialoga" con la red, separando el sistema en una parte de control y en una parte operativa.

Las redes de Petri temporizadas son redes no autónomas, cuyo entorno suministra una referencia de tiempo común. Se utilizan para estudiar el comportamiento dinámico de los sistemas, teniendo en cuenta, únicamente, la duración de sus acciones ( $y$ no la manera en que transforman el estado del entorno). En este caso, la presencia de una marca en una plaza, controla una acción a la que se le asocia una duración. Ésta, que puede ser variable en el caso general, viene representada por una constante no negativa, asociada a la plaza que controla la acción.

Para tener en cuenta la duración de las acciones, se modifican las reglas de franqueo de las transiciones. Así, si una marca llega a una plaza " $p$ " en un instante " $t$ " y si a partir de esta plaza se controla una acción de duración
" $z$ ", esta marca no puede abandonar la plaza " $p$ " antes del instante " $t+z$ ".

A causa de ello se supone que las marcas en las redes temporizadas tienen dos estados: disponible e indisponible. El paso del estado disponible al indisponible se efectúa en el momento del franqueo de las transiciones, de manera que las marcas colocadas en las plazas de salida de una transición, después de su franqueo, se encuentran en el estado indisponible. Éste corresponde a la situación en que, por su sola presencia, una marca controla una acción que se está ejecutando. Las marcas pasan del estado indisponible al estado disponible en una plaza " $p$ ", a partir del momento en que la acción asociada a " $p$ " ha terminado. Entonces pueden utilizarse para el franqueo de las transiciones.

El funcionamiento de una red temporizada puede simularse, aplicando el principio de separación entre parte de control y parte operativa, de la siguiente manera: la parte operativa contiene un conjunto de contadores, sincronizados por un reloj común. Cada plaza de la red tiene asociado un contador en el entorno. La llegada de una marca a una plaza, inicializa el contador en un valor que corresponde a la duración de la acción controlada. Los contadores bajan en una unidad de tiempo en cada impulso de reloj, siempre que sus valores sean positivos. Cuando el valor de un contador es cero, se envía a la parte de control un aviso de fin de acción. Este aviso convierte en disponible las marcas que controlaba la acción.

\section{Aplicaciones de las redes de Petri}

Las redes de Petri son un método de análisis previo a la implementación de sistemas dinámicos. Pueden utilizarse para especificar, validar e implementar todo sistema discreto con evoluciones simultáneas, siendo recomendable su uso cuando estos sistemas se comunican con el exterior

Como posible dominio de aplicacion, puede citarse la implementacion de la dinámica de los sistemas de información, obien la gestion de producción. Se utilizan -desde hace ya algunos años- en la concepción de mecanismos de asignación de recursos y de procedimientos de sincronización de tareas en sistemas centralizados o repartidos, así como para la elaboración de cuadernos de carga de sistemas de control de procesos industriales.

Dentro de la construcción, aparte de las aplicaciones en la fabricación de materiales y en la prefabricación de elementos, se está trabajando actualmente en la estandarización del intercambio de información por vía electrónica entre los distintos participantes en el hecho constructivo (Vries, 95), utilizando las redes de Petri para modelar los sistemas

Durante el proyecto, las actividades se inician a la recepción de información. Esta información puede adoptar formas diversas, dependiendo de ellas la vía de comunica- 
ción a utilizar. Para los seres humanos, las formas de comunicación son: hablar y escuchar, escribir y leer, y dibujar e interpretar el dibujo. Ahora ha de incluirse la comunicación por medio de computadores. Al igual que en los otros casos, deben establecerse protocolos entre remitente y destinatario, para que ambos se entiendan debidamente.

Siempre que haya intercambio de información entre dos partes, el protocolo debe hacer posible que tanto el remitente como el destinatario puedan interpretar la información, que la interpreten de la misma manera, que se intercambie la información requerida y que ésta sea la correcta.

En el proceso constructivo, se establecen acuerdos entre los participantes sobre las actividades que cada uno llevará a cabo. Cuando se utilizan ordenadores, el intercambio de datos o de mensajes entre participantes que desarrollan distintas actividades se hace de forma electrónica. Para hacer posible esta labor, una informacion organizada y eficiente debe ser facilitada a los participantes mediante un procedimiento adecuado, lo que implica la necesidad de una aproximación sistemática. Es decir, se requiere de un sistema de información, entendido como la red que proporciona los medios, a través de los cuales la informacion y los datos son procesados y transmitidos desde su origen a los diferentes usuarios y cuyo objetivo es proporcionar a los participantes, en todas las etapas del proceso arquitectónico, un sistema común y de fácil operabilidad, adaptable a las nuevas tecnologías utilizadas en la industria de la construcción.

\section{Conclusiones}

Las redes de Petri son un elemento de modelización y análisis previo de sistemas dinámicos, que conjuga el uso de técnicas gráficas y analíticas. Pueden utilizarse para especificar, validar e implementar todo sistema discreto con evoluciones simultáneas, siendo recomendable su uso cuando estos sistemas se comunican con el exterior.

Como posible dominio de aplicación se puede citar la implementación de la dinámica de los sistemas de información, o bien la gestión de producción. Son una herramienta muy importante, que debería ser conocida por cualquier experto en ciencias de la computación.

Dentro del campo de la construcción, sus aplicaciones más claras están en la fabricación de materiales y elementos y en el intercambio de mensajes electrónicos entre los distintos participantes en el proceso constructivo.

Se describen las redes de Petri básicas, así como distintas extensiones de las redes de Petri, tales como la utilización de arcosinhibidores o del marcado de plazas, la priorización de transiciones, las redes multicoloreadas y las redes temporizadas.

\section{BIBLIOGRAFÍA}

(1) C.A. PETRI: "Kommunication mit automaten", $\mathrm{Ph}$. D.Disertation, Universidad de Bonn, 1962.

(2) G. V. BRAMS: "Las redes de Petri. Teoría y práctica", Vol. 1,2. Editorial Masson, Barcelona, 1986.

(3) M. SILVA: "Las redes de Petri: En la automática y la informática. Editorial AC, Madrid, 1985.

(4) J. L. PETERSON: "Petri nets theory and the modelling of systems". Editorial Prentice-Hall, USA, 1981.

(5) W. REISIG: "Petrinets an introduction". Editorial Springer Verlag, Berlin, 1982.

(6) K. JENSEN y G.ROZENBERG: "High-level Petri nets". Editorial Springer-Verlag, Berlin, 1991.

(7) G. ROZEMBERG: "Advances in Petri nets". Editorial Springer-Verlag, Berlin, 1990.

(8) Proccedings of "International Workshop on Petri nets and Performance Models", 1985.

(9) M. HACK: "Decidability questions for Petri nets". Edit. MAC Techm., Report 161, M.I.T., USA, 1976.
(10) P.HUBER, A.M. JENSEN, L.O. JEPREN y K. JENSEN: "Reachibility trees for high level Petri nets". Theorical Computer Science 45, 1986, pp 261-292.

(11) T. AGERWALA y M. FLYNN: "Comments on capabilities, limitations and correctness of Petri nets". First annual Simposyum on Computer Architecture, Florida, 1979, pp 81-86.

(12) P. HUBER, K. JENSEN y R.M. SHAPIRO: "Hierchies in coloured Petrinets". Computer Science, vol 483. Editorial Springer-Verlag, Berlin, 1990, pp 342-416.

(13) G. CHIOLA, C. DUTHERLET y G. FRANCES: On well formed coloured nets and their symbolic reachibility graphs. 11th Int. Conference on Applications and theory of Petri nets, Paris, 1990.

(14) C. LIN y D.C. MARINESCU: "Stocahastic high-level Petri nets and applications". IEEE Transactions on computer vol. 37, 7,1988, pp 815-825.

(15) A. A. DESROCHERS: "Applications of Petri nets in manufacturing systems: modeling, control and performance analysis”. Editorial IEEE Press, N.York, 1995. 
(16) A. S. TANENBAUM: "Computer networks". Editorial Prentice-Hall International, USA, 1996.

(17) B. VRIES de y L.J. SOMERS: "Message exchange in the building industry". Automation in Construction Vol. 4, 1995, pp 91-100.
(18) F. DICESARE et al.: "Practice of Petrinets in manufacturing", Editorial Chapman-Hall, Londres, 1993.

(19) A. RECUERO , M. ÁlVAREZ, M. CALDERÓN e I. MORENO: "Análisis de redes de Petri mediante su árbol de cobertura". Métodos Numéricos para Cálculo y Diseño en ingeniería (en prensa).

\section{Publicaciones del Instituto Eduardo Torroja-CSIC}

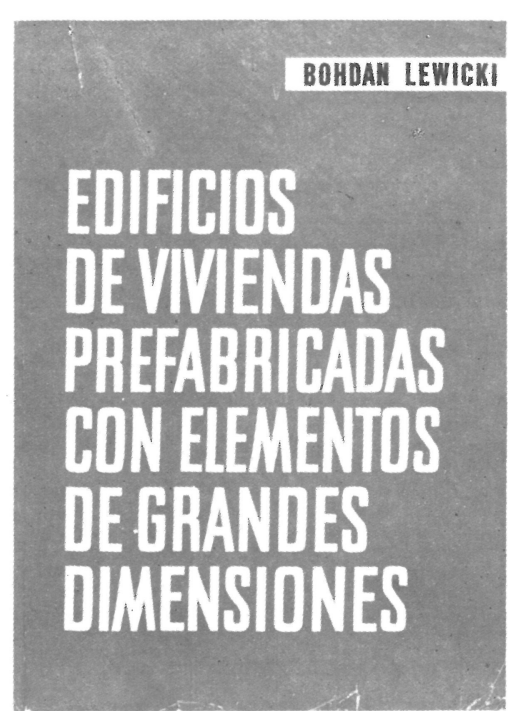

Bohdan Lewicki

Este libro trata de los problemas relativos a la construcción de los edificios de viviendas o publicos realizados con elementos prefabricados de grandes dimensiones. Se han estudiado los problemas de arriostramiento, asi como los que plantea la resistencia de los elementos y de la estructura; se han examinado las cuestiones de orden higrotérmico, acústico y de resistencia al fuego; también se ha profundizado en el estudio de la estanquidad de los muros exteriores y de las juntas.

La obra incluye numerosas ilustraciones que dan detalles de diversas soluciones, asi como ejemplos de cálculo, tablas de valores numéricos, diagramas y ábacos.

Un volumen encuadernado en tela, de $24 \times 17 \mathrm{~cm}$, compuesto de 616 págs.
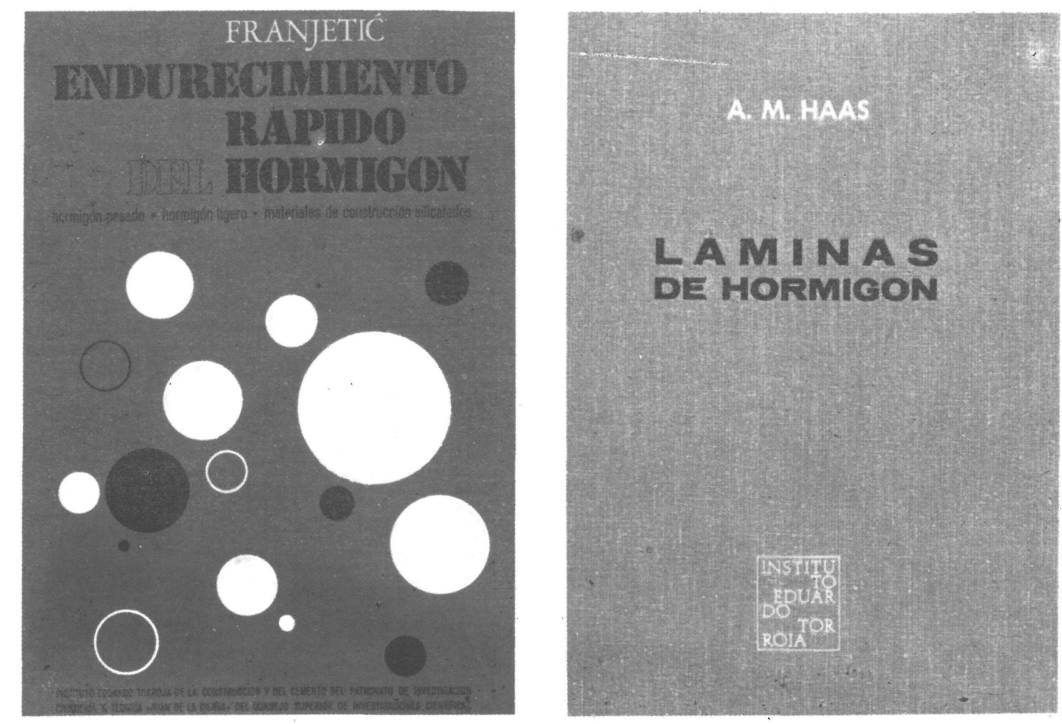

\section{Zorislav Franjetić}

En la obra de Franjetić se expone de una forma minuciosa, ordenada y sistemática, todo un cuerpo de doctrina que reúne el conocimiento actual sobre el endurecimiento rápido del hormigón. Parte el autor de los principios básicos y llega a las últimas consecuencias y realidades técnicas y economicas.

Es una obra de consulta, tanto para el investigador sobre la materia, como para e proyectista y el realizador y montador de plantas e instalaciones y equipos de curado y endurecimiento rápido.

Un volumen encuadernado en cartóné, de $17 \times 24,5 \mathrm{~cm}$, compuesto de 385 págs. 110 figuras y 10 tablas.

\section{A. M. Haas}

Al escribir este libro el autor intento poner a disposición de los estudiantes y de los ingenieros unos conocimientos prácticos, adecuados para servir de guia en el diseño y construcción de láminas delgadas de hormigón.

El autor está convencido de que el éxito en el diseño de una lámina exige, por parte del proyectista, un examen de las tres fases por las que pasa la materialización de la lámina: el diseñc, el análisis estructural y la construcción de la estructura.

Un volumen encuadernado en tela, de $17 \times 24,5 \mathrm{~cm}$, compuesto de 420 págs., 141 figuras, 22 fotografias y 6 tablas. 